\title{
A classification model base on Differential Evolution algorithm
}

\author{
Zhehuang Huang \\ (School of Mathematics Sciences, Huaqiao University, 362021, Quanzhou, China)
}

\begin{abstract}
Differential evolution algorithm is a heuristic global optimization technique based on population which is easy to understand and implement. In this paper we proposed a classification model base on differential evolution algorithm. The proposed model provides a good practicability and promising future for pattern identification.
\end{abstract}

Keywords: classification model, differential evolution, Intelligent Optimization.

\section{INTRODUCTION}

Differential Evolution (DE) is a population-based evolutionary algorithm proposed by the Storn and Price in 1997[1,2]. It is the first attempt to use the vector difference for shuffling vector populations, so as to solve the problem of polynomials adaptability [3, 4].Through cooperation and competition among individuals within populations, DE can address the optimization problems, which is essentially based on real-coded evolutionary algorithm with ensuring quality thinking. It can achieve better performance for a variety of test problems, and has become one of the hotspots in recent years.

Because its advantages in the continuous domain optimization[5,6], DE has been widely used, and sparked a research upsurge in the field of evolutionary algorithm [7,8]. It has the following advantages:

(1) Versatility and does not rely on the information of problem;

(2) Easy to implement;

(3) The optimal solution has a memory capacity of the individual;

(4) Cooperative search and can effectively use local information and global information;

(5) Easy to combine with other algorithms to construct an effective hybrid algorithm.

Of course, DE algorithm also has several drawbacks, such as:

(1) Local search capability is weak;

(2) Search performance of algorithm has a certain dependence on the parameters;

(3) Search performance needs to be strengthened and difficult to get global optimal solution within a limited time;

(4) Lack of practical guidelines.

In this paper, we proposed a new classification model based on DE algorithm. The proposed model has good global convergence which is better than the traditional intelligent optimization algorithm.

This paper is organized as follows. The background of DE is described in section 2. In section 3,a classification model base on DE is presented. Section 4 concludes the paper.

\section{BACKGROUND OF DE}

Suppose that the individual is represented as $x_{i}=\left[x_{i, 1}, x_{i, 2} \ldots, x_{i, d}\right]$. The new population can be generated by the following formula:.

$x_{i, k}(0)=l_{k}+\operatorname{rand}() \bullet\left(u_{k}-l_{k}\right), k=1,2, \ldots, d, i=1,2, \ldots, N$.

\section{Mutation operation}

An individual can be generated by the following formula:

$t_{i}(g)=x_{r 1}(g)+F \bullet\left[x_{r 2}(g)-x_{r 3}(g)\right]$,

Here $r_{1}, r_{2}, r_{3}$ are random numbers generated within the interval [1, N], variation factor $F$ is a real number of the interval [0,2], it control amplification degree of the differentia variable $x_{r 2}(g)-x_{r 3}(g)$. g represents the iterations of evolution.

\section{Crossover operation}

In difference algorithm, the cross operate is introduced to the diversity of the new population. According to the crossover strategy, the old 
${ }^{1}$ and new individual exchange part of the code to form a new individual ${ }_{1}$. New individuals can be represented as follow:

$$
v_{i, j}(g)=\left\{\begin{array}{l}
t_{i, j}(g), r<C R \text { or } j=r n d \\
x_{i, j}(g), \text { otherwise }
\end{array}\right.
$$

Where $r$ nd is uniformly distributed in the interval $[0,1], C R$ is crossover probability in the interval $[0,1]$.

\section{Selection operation}

Selection operation is greedy strategy, the candidate individual generated from mutation and crossover operation competition with target individual.

$x_{i}(g+1)=\left\{\begin{array}{l}t_{i}(g), f\left[t_{i}(g)\right]<f\left[x_{i}(g)\right] \\ x_{i}(g), \text { otherwise }\end{array}\right.$

The flow chart of differential evolution algorithm is shown in Figure 1.

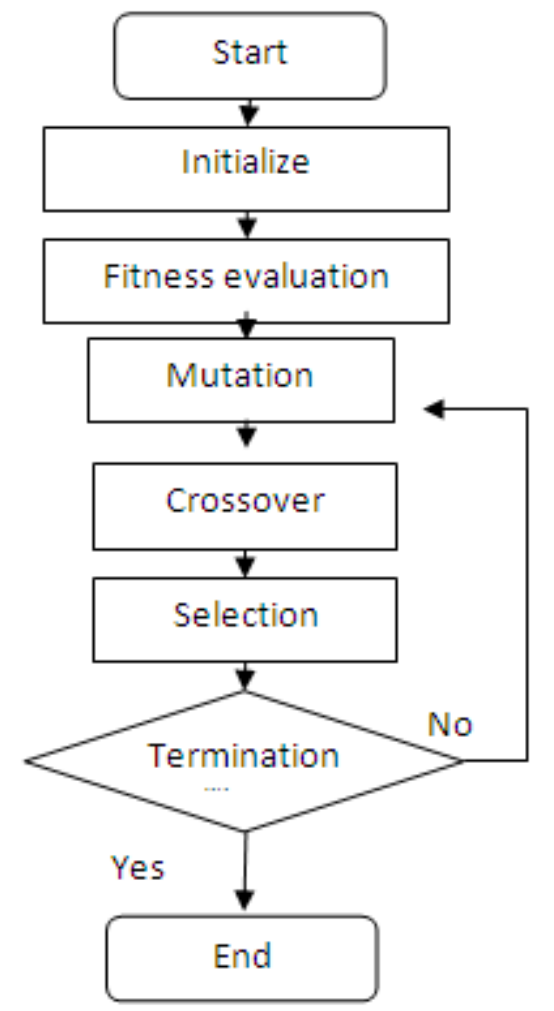

Fig.1. Flow chart of difference evolution algorithm.

The basic differential evolution (DE) algorithm is shown as Algorithm 1.

1) Initialize the population;

3) Produce a new generation of individual:

a) Mutation operation;

b) Crossover operation;

c) Selection operation.

4) Until the termination criterion is met. Algorithm 1. The differential evolution algorithm

\section{CLASSIFICATION ALGORITHM BASE ON DE}

\section{Feature engineering}

The acquisition of features is a key issue, we can extract features based on the actual problem.

The construction of feature space is shown as figure 2 . 


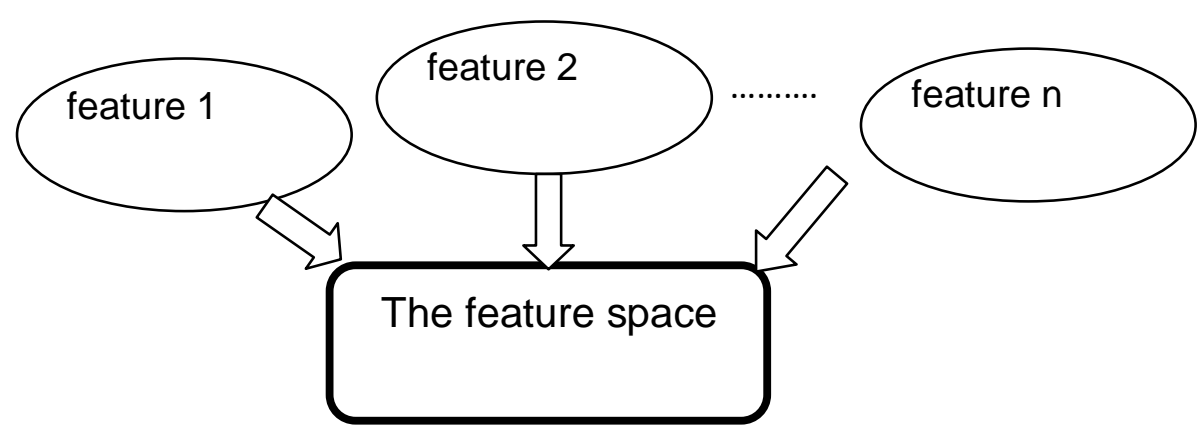

Fig.2. The construction of feature space

\section{Classification model}

Suppose $X=\left(x_{1}, x_{2} \cdots x_{n}\right), x_{i}=\left(\right.$ feature $i_{-} \min$, feature $\left.i_{-} \max \right)$.

if featurei_min $<x_{i}<$ featurei_max, $(i=1,2 \cdots n)$, then class_ $\mathrm{x}$

We use the differential evolution algorithm to get the optimal solution in the parameter space. The classification model base on DE is shown as figure 3 .

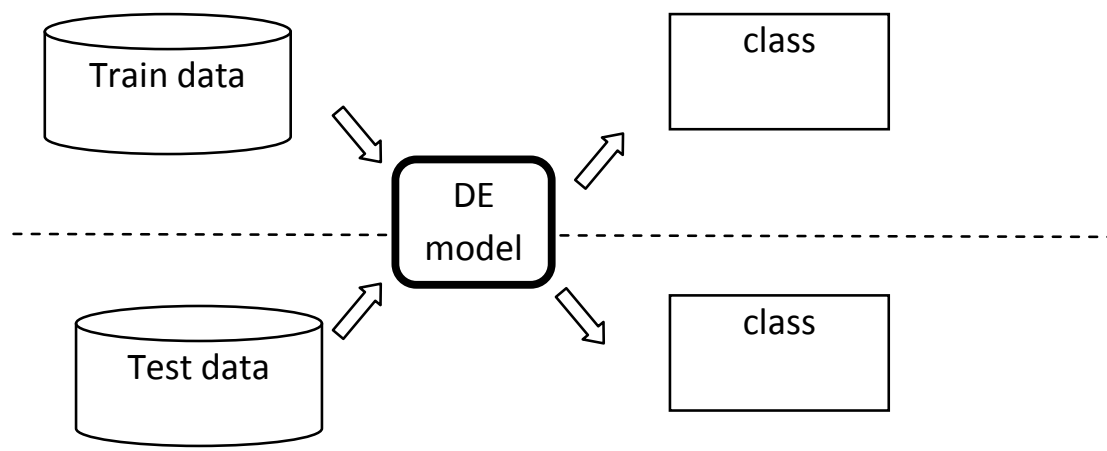

Fig.3. classification model based on DE

IV. CONCLUSION

In this paper, we proposed a classification model base on differential evolution algorithm which provides a good practicability for pattern classification. We will apply it to other areas.

\section{ACKNOWLEDGEMENTS}

This work was supported by the science and technology project of Quanzhou (Grant No.2012Z91).

\section{REFERENCES}

[1] Storn R,Price K, Differential evolution-a simple and efficient heuristic for global optimization over continuous spaces, Journal of Global Optimization, 11,1997,341-359.

[2] Storn R, Price K,Differential evolution for multi-objective optimization,Evolutionary Computation, 4,2003,8-12.

[3] Omran M G G, Engelbrecht A P. Self-Adaptive Differential Evolution Methods for Unsupervised Image Classification,Proc of the IEEE Conference on Cybernetics and Intelligent Systems.Bangkok, Thailand,2006,1-6.

[4] Yang Shiwen,Gan Y B, Qing Anyong. Sideband Suppression in Time-Modulated Linear Array by the Differential Evolution Algorithm. IEEEE Trans on Antennas and Wireless Propagation Letters, 1(1),2002, 173-175.

[5] Wu Lianghong, Wang Yaonan, Yuan Xiaofang,et al. Differential Evolution Algorithm with Adaptive Second Mutation,Chinese journal of control and decision , 21(8),2006,898-902.

[6] Tasgetiren M F, Suganthan P N, Chua T J, et al. Differential evolution algorithms for the generalized assignment problem,IEEE Congress on Evolutionary Computation, CEC 2009, 2009,2606-2613.

[7] Zhang Wen-gong, Chen Hua-ping, Lu Di, et al. A novel differential evolution algorithm for a single batch processing machine with non-identical job size, The Fourth International Conference on Natural Computation, 2008,447-451.

[8] Brest J, Greiner S, Boskovic B, et al. Self-adapting control parameters in differential evolution: A comparative study on numerical benchmark problems, IEEE Transactions on Evolutionary Computation, 10(6),2006,646-657. 